BMJ Open

Diabetes

Research

\& Care

\section{Timing of delivery in women with pre- pregnancy diabetes mellitus: a population-based study}

To cite: Brown M, Melamed N, Murray-Davis B, et al. Timing of delivery in women with prepregnancy diabetes mellitus: a population-based study. BMJ Open Diab Res Care 2019;7:e000758. doi:10.1136/ bmjdrc-2019-000758

- Additional material is published online only. To view please visit the journal online (http://dx.doi.org/10.1136/ bmjdrc-2019-000758).

Received 17 July 2019 Revised 31 October 2019 Accepted 25 November 2019

Check for updates

(c) Author(s) (or their employer(s)) 2019. Re-use permitted under CC BY-NC. No commercial re-use. See rights and permissions. Published by BMJ.

For numbered affiliations see end of article.

Correspondence to Dr Howard Berger; howard.berger@unityhealth.to

\section{ABSTRACT}

Objectives Controversy exists about the timing of delivery of women with pre-pregnancy type 1 and 2 diabetes mellitus (PDM). This study aims to compare maternal and neonatal outcomes after induction of labor (IOL) at 38 weeks' gestation versus expectant management from 39 weeks onward.

Research design and methods This was a retrospective population-based cohort study using data from the Better Outcomes Registry and Network in Ontario Canada. Included were all women with PDM, who had a singleton hospital birth at $\geq 38^{0 / 7}$ weeks' gestation from 2012 to 2017. Maternal and perinatal outcomes were compared between 937 pregnancies that underwent IOL at $38^{0 / 7}-38^{6 / 7}$ weeks ('38-IOL group') versus 1276 pregnancies expectantly managed resulting in a birth at $\geq 39^{0 / 7}$ weeks ('39-Exp group'). The primary outcome was all-cause cesarean delivery. Multivariable modified Poisson regression was performed to generate adjusted relative risks (aRR) and 95\% Cls, adjusted for parity, maternal age, pre-pregnancy body mass index and PDM type. Other outcomes included instrumental delivery, neonatal intensive care unit (NICU) admission, and newborn metabolic disturbances.

Results Cesarean delivery occurred in 269 women $(28.7 \%)$ in the $38-$ IOL group versus 333 women $(26.1 \%)$ in the 39-Exp group-aRR 1.07 (95\% $\mathrm{Cl} 0.94$ to 1.22$)$. The respective rates of instrumental delivery were $11.2 \%$ and $10.2 \%$ (aRR $1.25,95 \% \mathrm{Cl} 0.98$ to 1.61). NICU admission was more common in the 38 -IOL group $(27.6 \%)$ than in the 39-Exp group (16.8\%) (aRR 1.61, 95\% $\mathrm{Cl} 1.36$ to $1.90)$, as were jaundice requiring phototherapy $(12.4 \%$ vs $6.2 \%$ ) (aRR $1.93,95 \% \mathrm{Cl} 1.46$ to 2.57 ) and newborn hypoglycemia ( $27.3 \%$ vs $14.7 \%$ ) (aRR $1.74,95 \% \mathrm{Cl} 1.46$ to 2.07).

Conclusion In pregnant women with PDM, IOL at $38^{0 / 7}-38^{6 / 7}$ weeks was not associated with a higher risk of cesarean delivery, compared with expectant management, but was associated with a higher risk of certain adverse neonatal outcomes.

\section{INTRODUCTION}

Pre-pregnancy diabetes mellitus (PDM)type 1 or type $2 \mathrm{DM}$ preceding conception-is increasing in prevalence, with important adverse outcomes for mother

\section{Significance of this study}

What is already known about this subject?

- Pregnancies complicated by pre-pregnancy type 1 and 2 diabetes mellitus (PDM) are at increased risk of adverse perinatal outcomes.

- While induction of labor before 40 weeks' gestation can potentially reduce the rate of certain adverse prenatal outcomes, early delivery is also associated with an increase in neonatal complications.

What are the new findings?

- In women with PDM, induction of labor between $38^{0 / 7}$ and $38^{6 / 7}$ was not associated with an increased cesarean section rate compared with expectant management beyond 39 weeks.

- In women with PDM, induction of labor between $38^{0 / 7}$ and $38^{6 / 7}$ was associated with an increased rate of neonatal intensive care admission, jaundice and hypoglycemia compared with expectant management beyond 39 weeks.

How might these results change the focus of research or clinical practice?

- This study provides additional evidence regarding timing of delivery in women with PDM that can be used in formulating clinical practice guidelines and as the basis for future prospective randomized trials.

and newborn. ${ }^{1}{ }^{2}$ Despite improved glycemic control, and a declining rate of some congenital anomalies, ${ }^{2}{ }^{3}$ perinatal mortality, preterm birth, large for gestational age (LGA) birth weight, shoulder dystocia and stillbirth remain high. ${ }^{4}$ Furthermore, women with PDM experience higher rates of preeclampsia, and are more likely to deliver by cesarean section (CS), compared with women without PDM. ${ }^{356}$

Induction of labor (IOL) at 38-40 weeks' gestation has been endorsed as a part of the management of a pregnancy affected by PDM to prevent stillbirth, and to decrease macrosomia-related complications such as shoulder dystocia, anal sphincter 
injuries, birth trauma, and possibly, the need for cesarean delivery. ${ }^{7-10}$ Routine IOL may also avoid the development of maternal complications, such as pre-eclampsia, which is more common in a pregnancy complicated by PDM. ${ }^{6}$ However, routine IOL prior to 39 weeks' gestation may place a neonate at higher risk of complications related to early term delivery, and may also increase the rate of $\mathrm{CS},{ }^{11-13}$ although the latter notion has been recently challenged by data from studies in non-PDM populations. ${ }^{14-16}$

Data to support the optimal timing for IOL for women with PDM are lacking. Expert guidelines supporting routine IOL are based on small studies, and extrapolated from outcome data in pregnancies complicated by both gestational diabetes mellitus (GDM) and PDM. $^{7} 1718$ Recently published data suggest that, in women with GDM, routine IOL at 38 or 39 weeks is associated with a lower risk of cesarean delivery compared with expectant management but has a higher rate of neonatal intensive care unit (NICU) admission. ${ }^{19}$ Studies that specifically address the timing of IOL for women with diabetes focused on women with GDM, ${ }^{20-23}$ which may not be generalizable to women with PDM, who have a higher risk of complications. ${ }^{24}$

The aim of the current study was to compare maternal and neonatal outcomes in women with PDM induced at $38^{0 / 7}-38^{6 / 7}$ weeks' gestation versus women with PDM expectantly managed, and who remained undelivered at $39^{0 / 7}$ weeks' gestation.

\section{RESEARCH DESIGN AND METHODS}

Study setting

This population-based cohort study included all women with PDM in the province of Ontario, Canada. Ontario is the most populous province in Canada with a population of 14 million people ${ }^{25}$ and all residents receive universal health coverage under the government-funded provincial health insurance plan (OHIP: Ontario Health Insurance Plan). During the study period there were no national or provincial guidelines dictating timing of delivery in women with PDM, only broad suggested IOL guidelines from international societies. ${ }^{8926}$

\section{Data sources}

Data were obtained from the Better Outcomes Registry and Network Information System (BIS) (http://www. bornontario.ca/en/about-born), linked to the Canadian Institute of Health Information-Discharge Abstract Database (DAD). The BIS is a province-wide registry of all hospital and home births in Ontario, and contains details about each pregnancy and birth, including obstetrical, maternal and newborn measures. BIS data are collected through a variety of mechanisms including HL7, batch upload, and manual entry. Agreement between BIS and DAD has been validated internally and reveals $92.1 \%$ agreement for IOL and $99.8 \%$ agreement for mode of delivery. The Better Outcomes Registry and Network's (BORN) data quality framework includes processes for regular data validation, quality checks, and training for individuals entering and using the data to support a high level of data quality (http://www.bornontario.ca/en/ data/).

The DAD contains a set of validated diagnostic codes from the International Statistical Classification of Diseases and Related Health Problems, 10th Revision, Canadian version, as well as intervention codes from the Canadian Classifications of Health Intervention for all in-hospital deliveries. The linkage between the BIS and the DAD was done to ensure the highest degree of capture of maternal conditions preceding pregnancy, including PDM, and neonatal outcomes occurring in the NICU, which may not be fully detailed within the BIS. All diagnostic and procedural codes, along with definitions used to identify the cohort, characteristics, exposure and outcome variables are listed in online supplementary appendix S1.

\section{Study population}

The study population comprised all singleton pregnancies with PDM who were undelivered at $\geq 38^{0 / 7}$ weeks' gestation between 1 April 2012 and 31 March 2017. PDM was defined as a pre-pregnancy diagnosis of type 1 or type 2 diabetes. ${ }^{27}$ To create a low-risk obstetric cohort of women with PDM, who would be eligible for a vaginal birth, women with the following conditions were excluded: gestational age at birth $\geq 42^{0 / 7}$ weeks, non-vertex presentation, placenta previa, previous CS, major fetal anomaly, chronic hypertension, chronic renal disease, complications of diabetes (eg, retinopathy, renal dysfunction), congenital or acquired cardiac disease, pulmonary hypertension, cystic fibrosis, pulmonary embolism, systemic lupus erythematosus, hemophilia or sickle cell disease. Women with PDM who were diagnosed with gestational hypertension or pre-eclampsia before $38^{0 / 7}$ weeks were also excluded. A pragmatic decision was made to include only women who delivered after 38 weeks of gestation since in our population, women would not be routinely induced prior to 38 weeks of gestation without an additional cause or comorbid state.

\section{Exposure}

The cohort was divided into two exposure groups, to mimic the real-life dilemma faced by a healthcare provider: whether to induce at 38 weeks' gestation or expectantly manage until at least $39^{0 / 7}$ weeks. Women who underwent IOL between $38^{0 / 7}$ and $38^{6 / 7}$ weeks ('38-IOL group') were compared with those expectantly managed and who remained undelivered by $39^{0 / 7}$ weeks (ie, delivered anytime between $39+0$ weeks and $41+6$ weeks of gestation) ('39-Exp group'). Outcomes from all modes of delivery (vaginal birth with or without IOL, operative delivery, CS) from women in the 39-Exp group were included in analysis. All indications for IOL were included in the 39-Exp group.

For the 38-IOL group, in order to simulate routine IOL, we included women in whom the only indication for IOL was PDM, or a state related to PDM, such as fetal 
macrosomia; hence, women with an indication for IOL, such as gestational hypertension or pre-eclampsia, were excluded from the 38-IOL group. Similarly, as spontaneous labor is not a decision option for the clinician, we excluded women who went into spontaneous labor between $38^{0 / 7}$ and $38^{6 / 7}$.

\section{Outcomes}

The primary outcome was the relative rate of CS between groups. Secondary maternal outcomes included instrumental delivery, shoulder dystocia, postpartum hemorrhage, intrapartum fever, and anal sphincter injury (third or fourth-degree perineal lacerations). Secondary neonatal outcomes included NICU admission, hypoglycemia, jaundice requiring phototherapy, respiratory morbidity and a neonatal composite morbidity outcome. Neonatal respiratory morbidity was defined as any of the following: need for respiratory support in the form of continuous positive airway pressure or mechanical ventilation, or a diagnosis of transient tachypnea of the newborn or respiratory distress syndrome. The neonatal morbidity composite was defined as the presence of any of the following: perinatal mortality (stillbirth or neonatal death), 5 min Apgar score $<7$, admission to the NICU, hypoglycemia, jaundice requiring phototherapy, or neonatal respiratory morbidity. The 5 min Apgar score of $<7$ was chosen due to the documented increased risks of cerebral palsy and epilepsy that are seen in this range. ${ }^{28}$

\section{Statistical analysis}

Descriptive statistics compared the characteristics of women in the 38-IOL group and the 39-Exp group, using standardized differences to minimize the confounding effect of sample size. A standardized difference over 0.10 was considered clinically important. ${ }^{29}$ An a priori feasibility analysis was conducted to determine minimal sample size. With a power of $80 \%$ and using a two-sided alpha of 0.05 , we would require 626 women in the 38 -IOL group and 245 women in the 39-Exp group to detect a $13 \%$ absolute difference in our primary outcome.

Multivariable modified Poisson regression models, with robust error variance, were performed to calculate crude and adjusted relative risks (aRR) with $95 \%$ CIs, with the 39-Exp group as the referent. ${ }^{30}$ Models were adjusted for maternal age (continuous), pre-pregnancy body mass index (BMI) (continuous) and type of diabetes (ie, type 1 or type 2). As measures of glycemic control were not available in the data sets, a second model further adjusted for LGA birth weight greater than the 90th percentile (LGA), as a surrogate marker of glycemic control in pregnancy. An additional analysis examined the main model stratified by nulliparous and parous women.

Two-sided $p$ values were set at $<0.05$. All analyses were conducted with Statistical Analysis Software V.9.4.

\section{RESULTS}

Between April 2012 and March 2017, a total of 668245 women had a singleton hospital birth in Ontario, of whom
7558 (1.1\%) had PDM (figure 1). Among the latter, 2945 women $(40.0 \%)$ met the inclusion criteria. Of these, 937 were in the final 38-IOL group, after excluding 218 women for IOL for reasons other than PDM or macrosomia. There were 514 women who were excluded as they delivered between $38+0$ and $38+6$ weeks without undergoing IOL. A total of 1276 women were therefore included in the 39-Exp group. Those in the 38-IOL group had a higher BMI, were more likely to have type $1 \mathrm{DM}$, and were more likely to be on insulin treatment if they had type 2DM (table 1).

In the univariate analysis (online supplementary table S1) there was no significant difference in the rate of cesarean delivery ( $28.7 \%$ vs $26.1 \%$ (standardized difference 0.06$)$ ), or instrumental delivery (11.2\% vs $10.2 \%$ (standardized difference 0.03)), in the 38-IOL group compared with the 39-Exp group. The rate of other pregnancy outcomes such as intrapartum fever, anal sphincter injury and postpartum hemorrhage did not differ between the 38-IOL group and 39-Exp group, although the absolute rate of these outcomes was low $(<5 \%)$. Women in the expectant management group were at risk of developing new-onset gestational hypertension and pre-eclampsia $(5.0 \%$ and $0.7 \%$, respectively). A total of $53.1 \%$ of the women in the 39-Exp group went on to undergo IOL after $39+0$ weeks' gestation. As per study design, the median gestational age at delivery was lower in the 38-IOL group than in the 39-Exp group (38.3 (38.1-38.6) vs 39.6 (39.1-40.1)). Infants of women in the 38-IOL group were more likely to have a birth weight over the 90 th percentile $(27.3 \%$ vs $15.8 \%$ (standardized difference 0.28$)$ ), jaundice requiring phototherapy (12.4\% vs $6.2 \%$ (standardized difference 0.21$)$ ), hypoglycemia (27.3\% vs $14.7 \%$ (standardized difference 0.31$)$ ), and admission to the NICU (27.6\% vs $16.8 \%$ (standardized difference 0.26)) compared with the expectant management group. As such, the neonatal composite morbidity rate was significantly higher in the 38-IOL group $(47.9 \%$ vs $31.4 \%$ in the expectant group; standardized difference $0.34)$.

Multivariable analysis was conducted to adjust for potential confounding maternal factors that were found to be significant in the univariate analysis (online supplementary table S1) or have clinical significance when making a clinical decision on timing of IOL. These variables included maternal age, pre-pregnancy BMI, nulliparity and type of DM (type 1 vs type 2). There was no difference in the risk of cesarean delivery (aRR 1.07; $95 \%$ CI 0.94 to 1.22 ), instrumental delivery (aRR 1.25; $95 \%$ CI 0.98 to 1.61 ) or shoulder dystocia (aRR 1.35 ; $95 \%$ CI 0.9 to 1.97 ) in the 38 -IOL group compared with the 39-Exp group (table 2). The aRRs of most neonatal outcomes were increased in the 38-IOL group including an increased risk of NICU admission (aRR 1.61; 95\% CI 1.36 to 1.9 ), jaundice requiring phototherapy (aRR 1.93; $95 \%$ CI 1.46 to 2.57 ), hypoglycemia (aRR $1.74 ; 95 \%$ CI 1.46 to 2.07 ), and composite neonatal morbidity (aRR $1.50 ; 95 \%$ CI 1.34 to 1.67$)$ compared with the 39-Exp group. The addition of LGA birth weight $>90 \%$ to the multivariable analysis did not significantly alter these 


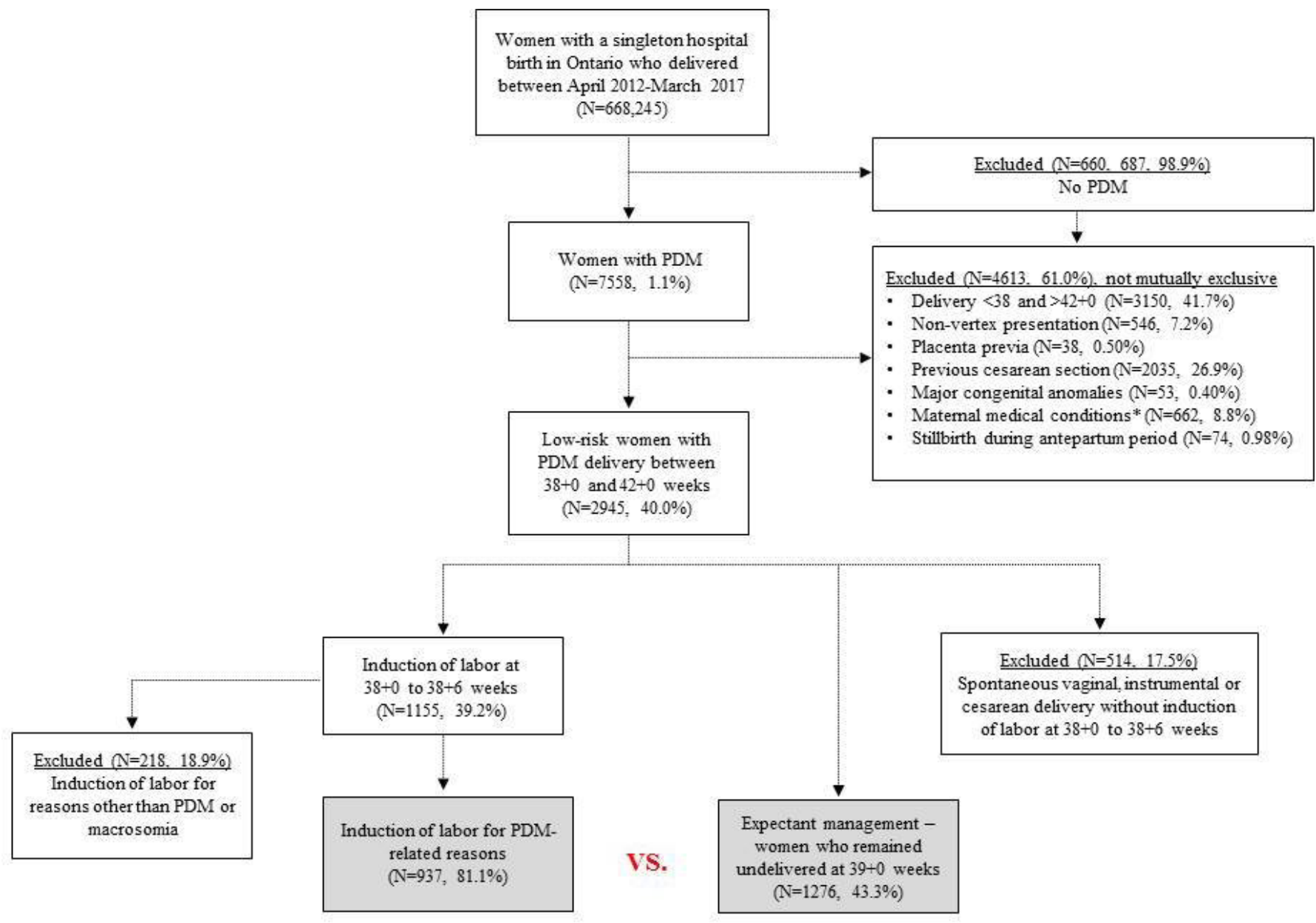

Selection of the induction and expectant management groups at 38 and 39 weeks. The asterisk refers to chronic medical conditions that include chronic hypertension, chronic renal disease, complications of diabetes (e.g retinopathy, renal dysfunction), cardiac disease (congenital or acquired), pulmonary disease (pulmonary hypertension, cystic fibrosis, pulmonary embolism), autoimmune conditions (e.g systemic lupus erythematosus) or hematologic diseases (hemophilia, sickle cell disease) that could influence a primary decision to induce labor at 38-39 weeks of gestation.

Figure 1 Flow diagram showing selection of study cohort, application of exclusion criteria, and formation of two exposure groups including the induction of labor between $38+0$ and $38+6$ weeks (38-IOL group) and the expectant management group with delivery beyond 39+0 weeks (39-Exp group). IOL, induction of labor; PDM, pre-pregnancy diabetes mellitus.

results. There were no cases of stillbirth or neonatal mortality in either group.

As IOL success is influenced by previous successful vaginal delivery, ${ }^{31-33}$ the adjusted analysis was further stratified by parity (online supplementary tables $\mathrm{S} 2$ and S3). The risk of cesarean delivery was not different in the IOL group compared with the expectant management group in nulliparous pregnancies (aRR 1.06; 95\% CI 0.93 to 1.21 ), or in multiparous pregnancies (aRR 0.93 ; $95 \%$ CI 0.63 to 1.36$)$.

\section{DISCUSSION}

Our study compared maternal and neonatal outcomes in a large cohort of women with PDM who underwent routine IOL between $38^{0 / 7}$ and $38^{6 / 7}$ weeks' gestation compared with those who were managed expectantly and delivered after $39^{0 / 7}$ weeks. Our findings are as follows: (1) there is no significant difference in the rate of CS, instrumental delivery or shoulder dystocia between the 38-IOL group and 39-Exp group, and this finding persisted after multivariable analysis; (2) neonates in the 38-IOL group had higher rates of NICU admission, jaundice requiring phototherapy and hypoglycemia when compared with the 39-Exp group; (3) there were no cases of stillbirth in the expectant group, but hypertensive disorders developed in $5.7 \%$ of women managed expectantly.

The main strengths of our study include the large sample size, the focus on an exclusive population of 'low risk' PDM, the ability to adjust for critical covariates and the uniform access of the population to publicly funded 
Table 1 Characteristics of the 38-IOL group versus 39-Exp group. All data are shown as a number (\%) unless otherwise indicated

\begin{tabular}{lccc}
\hline Characteristics & $\begin{array}{l}\text { 38-IOL group } \\
(\mathbf{n = 9 3 7 )}\end{array}$ & $\begin{array}{l}\text { 39-Exp group } \\
(\mathbf{n = 1 2 7 6 )}\end{array}$ & Standardized difference \\
\hline Mean (SD) maternal age (years) & $31.9(5.2)$ & $31.8(5.3)$ & 0.01 \\
\hline BMI $\left(\mathrm{kg} / \mathrm{m}^{2}\right)$ & & & \\
\hline Underweight $(<18.5)$ & $54(5.8)$ & $147(11.5 \%)$ & -0.21 \\
\hline Normal $(\leq 18.5$ to $<25)$ & $259(27.6)$ & $414(32.4)$ & -0.10 \\
\hline Overweight $(\leq 25$ to $<30)$ & $249(26.6)$ & $316(24.8)$ & 0.04 \\
\hline Obese $(>30)$ & $375(40.0)$ & $399(31.3)$ & 0.18 \\
\hline Missing & $40(4.3)$ & $95(7.4)$ & -0.14 \\
\hline Mean $(\mathrm{SD})$ BMI $\left(\mathrm{kg} / \mathrm{m}^{2}\right)$ & $28.1(7.4)$ & $29.9(7.6)$ & 0.23 \\
\hline Nulliparity & $451(48.1)$ & $647(50.7)$ & -0.05 \\
\hline Female newborn infant & $442(47.2)$ & $614(48.1)$ & -0.02 \\
\hline Type 1 diabetes mellitus & $316(33.7)$ & $315(24.7)$ & 0.2 \\
\hline Type 2 diabetes mellitus & $553(59.0)$ & $693(54.3)$ & 0.1 \\
\hline Insulin treatment & $438(79.2)$ & $321(46.3)$ & 0.72 \\
\hline Unknown type, diabetes mellitus & $68(7.3)$ & $268(21.0)$ & -0.4 \\
\hline
\end{tabular}

Data sources: Better Outcomes Registry and Network Ontario (2012-2017), Canadian Institute of Health Information-Discharge Abstract Database (CIHI-DAD; 2012-2015).

Cohort definition: Women who had a singleton birth who met the inclusion criteria.

BMI, body mass index; IOL, induction of labor.

prenatal care. The exclusion of women who went into spontaneous labor between $38^{0}$ and $38^{6}$ weeks of gestation, while initially counterintuitive, is consistent with the aim of this study to mimic the real-life decision process that the clinician is faced with when managing these patients.
Despite the large size of this cohort, it was still underpowered to detect very rare adverse outcomes, such as stillbirth and neonatal death, especially in this population of 'low-risk' PDM. The ability to comment on factors not adequately collected in the BORN database, such as information on adequacy of glycemic control, prenatal

Table 2 Outcomes among the 38-IOL group versus 39-Exp group

\begin{tabular}{|c|c|c|c|c|c|}
\hline \multirow[b]{2}{*}{ Outcome } & \multicolumn{2}{|c|}{ n (\%) with outcome } & \multirow[b]{2}{*}{$\begin{array}{l}\text { Crude RR } \\
(95 \% \mathrm{Cl})\end{array}$} & \multirow[b]{2}{*}{$\begin{array}{l}\text { Adjusted RR* } \\
(95 \% \mathrm{Cl})\end{array}$} & \multirow[b]{2}{*}{$\begin{array}{l}\text { Adjusted RR† } \\
(95 \% \mathrm{Cl})\end{array}$} \\
\hline & $\begin{array}{l}\text { 38-IOL group } \\
(n=937)\end{array}$ & $\begin{array}{l}\text { 39-Exp group } \\
(n=1276)\end{array}$ & & & \\
\hline \multicolumn{6}{|l|}{ Maternal } \\
\hline Cesarean delivery & $269(28.7)$ & $333(26.1)$ & $1.10(0.96$ to 1.26$)$ & 1.07 (0.94 to 1.22$)$ & 1.02 (0.90 to 1.16$)$ \\
\hline Instrumental delivery & $105(11.2)$ & $130(10.2)$ & $1.10(0.86$ to 1.40$)$ & $1.25(0.98$ to 1.61$)$ & 1.29 (1.00 to 1.66$)$ \\
\hline Shoulder dystocia & $57(6.1)$ & $52(4.1)$ & 1.49 (1.03 to 2.15$)$ & 1.35 (0.92 to 1.97$)$ & 1.05 (0.71 to 1.55$)$ \\
\hline \multicolumn{6}{|l|}{ Neonatal } \\
\hline Neonatal composite morbidity $\ddagger$ & $449(47.9)$ & $401(31.4)$ & 1.52 (1.37 to 1.69$)$ & 1.50 (1.34 to 1.67$)$ & 1.42 (1.27 to 1.59$)$ \\
\hline NICU admission & $259(27.6)$ & $215(16.8)$ & 1.64 (1.40 to 1.93$)$ & $1.61(1.36$ to 1.90$)$ & 1.50 (1.26 to 1.77$)$ \\
\hline Jaundice requiring phototherapy & $116(12.4)$ & $79(6.2)$ & 2.00 (1.52 to 2.63$)$ & $1.93(1.46$ to 2.57$)$ & 1.76 (1.32 to 2.33$)$ \\
\hline Hypoglycemia & $256(27.3)$ & $187(14.7)$ & 1.86 (1.57 to 2.21$)$ & 1.74 (1.46 to 2.07$)$ & 1.61 (1.35 to 1.93$)$ \\
\hline Respiratory morbidity§ & $114(12.2)$ & $135(10.6)$ & 1.15 (0.91 to 1.45$)$ & 1.11 (0.87 to 1.42$)$ & $1.02(0.80$ to 1.31$)$ \\
\hline
\end{tabular}

Cohort definition: Women who had a singleton birth who met the inclusion criteria.

Data sources: Better Outcomes Registry and Network Ontario (2012-2017), Canadian Institute of Health Information-Discharge Abstract

Database (CIHI-DAD; 2012-2015).

${ }^{*}$ Adjusted for maternal age, pre-pregnancy body mass index (BMI), nulliparity, and diabetes type.

†Adjusted for maternal age, pre-pregnancy BMI, nulliparity, diabetes type and large for gestational age (LGA) >90th percentile.

ҒDefined as the presence of any of the following: perinatal mortality (stillbirth or neonatal death), $5 \mathrm{~min}$ Apgar score $<7$, admission to the neonatal intensive care unit (NICU), hypoglycemia, jaundice requiring phototherapy, or neonatal respiratory morbidity.

$\S$ Refers to respiratory distress syndrome, transient tachypnea of the newborn infant, or need for respiratory support.

Exp, expectant management; IOL, induction of labor; RR, relative risk. 
ultrasound results, gestational weight gain, ethnicity or use of oral antihyperglycemic medications, was limited by the retrospective nature of this study. The use of LGA as a surrogate for glycemic control in the multivariate analysis assists in overcoming some of these limitations. It is recognized though, that the statistical analysis performed might not fully correct for the difference seen in the rate of insulin use in women with type 2 diabetes, a factor that could have influenced clinician decision-making and possibly some of the neonatal outcomes.

Unfortunately, this study was not powered to perform stratified analysis by type of medical therapy in women with type 2 diabetes. Stratification by type of PDM was not performed, as stillbirth rates generally do not differ between women with type 1 and 2 diabetes, and thus, it is might not be clinically relevant to stratify by diabetes type, with regard to timing of delivery. Of note, adding PDM subtype in the regression models did not alter the estimated risk of neonatal outcomes or CS.

The database was also found to have small proportions of missing data that are detailed in table 1, including a small proportion of patients in the database who were known to have PDM, but were not labelled as either type 1 or type $2 \mathrm{DM}$. It is also noted that the neonatal outcomes in this study are possibly under-reported, as capture of neonatal data was limited to all level 2 special care neonatal units (SCU) and only three out of eight level 3 SCUs in Ontario from 1 April 2015 to 31 March 2017.

A selection bias for IOL may also exist based on preferential IOL of women with higher Bishop scores, which cannot be ruled out in this study as preinduction Bishop score data were not available in the BORN database. While it is more likely that this bias affected the 38-IOL group, it should be noted that both the IOL group and the expectant management group had very similar proportions of nulliparous women $(48.1 \%$ and $50.7 \%$, respectively), who typically have lower Bishop scores compared with multiparous women. ${ }^{31}$ Indeed, the results of our study did not change in the secondary analysis that was restricted to nulliparous women. We therefore suspect that preinduction Bishop score is unlikely to be a significant bias in this study.

In the only published randomized controlled trial (RCT) comparing IOL with expectant management that included women with PDM, only 13 out of the 200 women studied had PDM. ${ }^{18}$ This study found no difference in the rate of CS or shoulder dystocia, and no difference in neonatal morbidity between IOL at 38 weeks compared with expectant management. Results from timing of delivery studies in GDM pregnancies, including a recent RCT, revealed findings similar to ours; Alberico et al randomized women with GDM by the International Association of Diabetes and Pregnancy Study Group criteria which included an estimated fetal weight of $<4000 \mathrm{~g}$ and an unfavorable cervix to IOL between 38 and 39 weeks of gestation versus expectant management until 41 weeks. ${ }^{23}$ Of the planned sample size of 1760 only 425 women were successfully randomized. No difference was seen in the CS rate between groups. The only neonatal outcome that was significantly different between groups was the rate of neonatal hyperbilirubinemia, which was increased in the IOL group.

Support for our results can also be found in studies of IOL in non-diabetic pregnancies. Boulvain et al randomized women with suspected LGA fetuses (mainly non-GDM) to IOL at $37-38^{+6}$ weeks versus expectant management until a maximum of 41 weeks' gestation. ${ }^{15}$ After successfully randomizing 822 women, no difference was found in the rate of CS (RR 0.89; 95\% CI 0.72 to 1.09) but higher rate of jaundice requiring phototherapy was noted ( $9 \%$ vs $3 \%$; $\mathrm{p}=0.0004$ ). In contrast to our findings, the Boulvain $e t$ als study noted the IOL group had a lower risk of shoulder dystocia (RR $0.32 ; 95 \%$ CI 0.12 to 0.85) compared with the expectant management group. In non-diabetic low-risk women, results from a recent large RCT also demonstrated that routine IOL before 40 weeks' gestation without a medical indication did not increase the odds of delivering by $\mathrm{CS} .{ }^{34}$ Retrospective cohort studies of low-risk women have also shown IOL before 41 weeks to be associated with increased odds of admission to the NICU. ${ }^{16}$

While these studies reflect outcomes in women without PDM, the uniformity of the results lends considerable support to our finding that routine IOL in women with PDM is not associated with an increase in the rate of CS delivery but will be associated with certain neonatal complications. Our study also found higher rates of adverse neonatal outcomes in the 38-IOL after controlling for LGA $>90$ th percentile as a marker of maternal glycemic control in pregnancy, which suggests that these outcomes are in fact related to earlier delivery rather than to a selection bias of pregnancies with poorly controlled diabetes.

Some findings in our study require further attention. When comparing study groups, we identified differences in the 38-IOL group compared with the 39-Exp group that likely reflect practice bias. Women in the 38-IOL group were more likely to have higher BMIs, to have type 1 diabetes, and to be on insulin treatment if they had type 2 diabetes compared with those in the 39-Exp group. It is hypothesized that caregivers are more likely to suggest IOL at 38 weeks in pregnancies with these factors, as they may confer higher risks of stillbirth, macrosomia and labor dystocia. We controlled for the possible confounding influence of these 'high risk' factors in our multivariable analysis and found that this did not change our principal findings. The fact that significantly more infants in the IOL group had birth weight over the 90th percentile, which might be a surrogate for inadequate glycemic control, also supports the notion that practitioners are preferentially choosing IOL at 38 weeks in pregnancies suspected of being at higher risk. The CS rate in our study population was low compared with that reported in other studies. ${ }^{35}$ This likely reflects the selective nature of our cohort, having 
excluded mothers with previous CS, chronic morbidities, and preterm deliveries.

Pregnancies complicated by PDM are less commonly encountered than those with GDM but confer higher maternal and neonatal risks. ${ }^{2}$ Guideline recommendations on timing of delivery vary widely and are poorly supported by high-quality evidence. ${ }^{8-10} 36$ Furthermore, while IOL before 40 weeks' gestation and more often before 39 weeks' gestation is commonly performed in pregnancies complicated by PDM, the effect of this intervention on the CS rate and neonatal outcomes is largely unknown. The argument in support of elective delivery before 39 weeks' gestation is largely fueled by the known increased risk of stillbirth in the PDM population. While it is reassuring that there were no cases of stillbirth in the expectant management group, this study was not powered for this relatively rare outcome and we cannot rule out that stillbirth might have occurred if women who underwent IOL at $<39$ weeks were instead managed expectantly. Recent data from the $\mathrm{UK}^{37}$ have shown that stillbirth rates have declined significantly despite little change in gestational age at delivery suggesting that factors such as improved surveillance or tighter glycemic control targets are perhaps more important than the timing of delivery.

Our results suggest that despite being at higher baseline risk, and delivering larger babies, women with PDM induced at 38 weeks did not have an increased risk of CS, shoulder dystocia or instrumental delivery when compared with expectant management. Whether this remains true at later gestational ages remains unclear. Although it is not possible to comment on the long-term clinical impact of these adverse neonatal outcomes, it is certainly an important factor to consider from a health services perspective as well as the short-term implications of NICU admission on parental bonding, breast feeding success and parental anxiety.

Further studies are needed to characterize how certain factors, such as pre-pregnancy BMI, glycemic control, sonographic parameters and type of PDM, could be used to guide decision-making on elective IOL in women with PDM. Ultimately, in the setting of pregnancies complicated by PDM, the decision to undergo elective IOL at less than 39 weeks' gestation will always involve balancing the risks of adverse neonatal outcomes with the benefits of avoiding future stillbirth or the development of new hypertensive complications. The answer to this question will ideally be answered by an appropriately powered RCT that will provide the much-needed level 1 evidence. Until this level of evidence is available, the results of this study can help guide clinical practice at the caregiver level, and provide needed evidence to assist in the creation of professional guidelines addressing the timing of delivery in women with PDM.
${ }^{2}$ Division of Maternal-Fetal Medicine, Department of Obstetrics and Gynecology, Sunnybrook Health Sciences Centre, Toronto, Ontario, Canada

${ }^{3}$ Department of Obstetrics and Gynecology, Midwifery Education Program, McMaster University, Hamilton, Ontario, Canada

${ }^{4}$ Better Outcomes Registry and Network, Children's Hospital of Eastern Ontario (CHEO), Toronto, Ontario, Canada

${ }^{5}$ Division of Maternal-Fetal Medicine, Department of Obstetrics and Gynecology, Unity Health Toronto, Toronto, Ontario, Canada

${ }^{6}$ Division of Maternal-Fetal Medicine, Departments of Obstetrics and Gynecology, Radiology and Health Research Methods, Evidence and Impact, McMaster University, Hamilton, Ontario, Canada

${ }^{7}$ Departments of Medicine and Obstetrics and Gynaecology, Unity Health Toronto, Toronto, Ontario, Canada

${ }^{8}$ Department of Obstetrics \& Gynaecology, Rotunda Hospital, Dublin, Ireland

Contributors $\mathrm{MB}$ and $\mathrm{HB}$ made substantial contributions to the conception, design, and interpretation of data for the work. NM, BMD, KM, JB, SDM, JGR and MG made substantial contributions to the conception and design of data for the work. HH made substantial contributions to the analysis of data for the work. All authors were involved in drafting the work or revising it critically for important intellectual content, had final approval of the version to be published, and were in agreement to be accountable for all aspects of the work in ensuring that questions related to the accuracy or integrity of any part of the work are appropriately investigated and resolved.

Funding This work was supported by the Canadian Institute of Health Research (CIHR) (Grant No 146442) and did not include an external peer review for scientific quality; Non-Communicable Diseases in Obstetrics: Improving Quality of Care and Maternal-Infant Outcomes Through an Obstetrical Research Network. Matched funding was provided by the Department of Obstetrics and Gynaecology at the University of Toronto, McMaster University, Sunnybrook Research Institute, and Providence St Joseph's and St Michael's Healthcare.

Competing interests None declared.

Patient consent for publication Not required.

Ethics approval Ethics approval to conduct this study was obtained from the St Michael's Hospital Research Ethics Board.

Provenance and peer review Not commissioned; externally peer reviewed.

Data availability statement Data are available from the Better Outcomes Registry and Network (https://www.bornontario.ca/en/data/access-the-born-informationsystem.aspx).

Open access This is an open access article distributed in accordance with the Creative Commons Attribution Non Commercial (CC BY-NC 4.0) license, which permits others to distribute, remix, adapt, build upon this work non-commercially, and license their derivative works on different terms, provided the original work is properly cited, appropriate credit is given, any changes made indicated, and the use is non-commercial. See: http://creativecommons.org/licenses/by-nc/4.0/.

ORCID iD

Howard Berger http://orcid.org/0000-0003-3842-213X

\section{REFERENCES}

1 Lawrence JM, Contreras R, Chen W, et al. Trends in the prevalence of preexisting diabetes and gestational diabetes mellitus among a racially/ethnically diverse population of pregnant women, 19992005. Diabetes Care 2008;31:899-904.

2 Feig DS, Hwee J, Shah BR, et al. Trends in incidence of diabetes in pregnancy and serious perinatal outcomes: a large, populationbased study in Ontario, Canada, 1996-2010. Diabetes Care 2014;37:1590-6.

3 Liu S, Rouleau J, León JA, et al. Impact of pre-pregnancy diabetes mellitus on congenital anomalies, Canada, 2002-2012. Health Promot Chronic Dis Prev Can 2015;35:79-84.

4 Melamed N, Hod M. Perinatal mortality in pregestational diabetes. Int J Gynaecol Obstet 2009;104 Suppl 1:S20-4.

5 Higgins M, Galvin D, McAuliffe F, et al. Pregnancy in women with type 1 and type 2 diabetes in Dublin. Ir J Med Sci 2011;180:469-73.

6 Feig DS, Razzaq A, Sykora K, et al. Trends in deliveries, prenatal care, and obstetrical complications in women with pregestational diabetes: a population-based study in Ontario, Canada, 1996-2001. Diabetes Care 2006;29:232-5. 
7 Lurie S, Insler V, Hagay ZJ. Induction of labor at 38 to 39 weeks of gestation reduces the incidence of shoulder dystocia in gestational diabetic patients class A2. Am J Perinatol 1996;13:293-6.

8 Berger H, Gagnon R, Sermer M, et al. Diabetes in pregnancy. $J$ Obstet Gynaecol Can 2016;38:e661:667-79.

9 ACoP B, Bulletin AP, ACOG Committee on Practice Bulletins. ACOG practice Bulletin. clinical management guidelines for ObstetricianGynecologists. number 60, March 2005. Pregestational diabetes mellitus. Obstet Gynecol 2005;105:675-85.

10 (NICE) NIfHaCE. diabetes in pregnancy: management of diabetes and its complications from preconception to the postnatal period. Clinical guideline NG3 2015.

11 Levine LD, Hirshberg A, Srinivas SK. Term induction of labor and risk of cesarean delivery by parity. J Matern Fetal Neonatal Med 2014;27:1232-6.

12 Moldéus K, Cheng YW, Wikström A-K, et al. Induction of labor versus expectant management of large-for-gestational-age infants in nulliparous women. PLoS One 2017;12:e0180748.

13 Caughey AB, Sundaram V, Kaimal AJ, et al. Maternal and neonatal outcomes of elective induction of labor. Evid Rep Technol Assess 2009:1-257.

14 Mishanina E, Rogozinska E, Thatthi T, et al. Use of labour induction and risk of cesarean delivery: a systematic review and metaanalysis. Can Med Assoc J 2014;186:665-73.

15 Boulvain M, Senat M-V, Perrotin F, et al. Induction of labour versus expectant management for large-for-date fetuses: a randomised controlled trial. Lancet 2015;385:2600-5.

16 Stock SJ, Ferguson E, Duffy A, et al. Outcomes of elective induction of labour compared with expectant management: population based study. BMJ 2012;344:e2838.

17 Greuter MJE, van Emmerik NMA, Wouters MGAJ, et al. Quality of guidelines on the management of diabetes in pregnancy: a systematic review. BMC Pregnancy Childbirth 2012;12:58.

18 Kjos SL, Henry OA, Montoro M, et al. Insulin-Requiring diabetes in pregnancy: a randomized trial of active induction of labor and expectant management. Am J Obstet Gynecol 1993;169:611-5.

19 Melamed N, Ray JG, Geary M, et al. Induction of labor before 40 weeks is associated with lower rate of cesarean delivery in women with gestational diabetes mellitus. Am J Obstet Gynecol 2016;214:364.e1-364.e8.

20 Witkop CT, Neale D, Wilson LM, et al. Active compared with expectant delivery management in women with gestational diabetes: a systematic review. Obstet Gynecol 2009;113:206-17.

21 Worda K, Bancher-Todesca D, Husslein P, et al. Randomized controlled trial of induction at 38 weeks versus 40 weeks gestation on maternal and infant outcomes in women with insulin-controlled gestational diabetes. Wien Klin Wochenschr 2017.
22 Lurie S, Matzkel A, Weissman A, et al. Outcome of pregnancy in class $A 1$ and $A 2$ gestational diabetic patients delivered beyond 40 weeks' gestation. Am J Perinatol 1992;9:484-8.

23 Alberico S, Erenbourg A, Hod M, et al. Immediate delivery or expectant management in gestational diabetes at term: the GINEXMAL randomised controlled trial. BJOG: Int J Obstet Gy 2017:124:669-77.

24 Lai FY, Johnson JA, Dover D, et al. Outcomes of singleton and twin pregnancies complicated by pre-existing diabetes and gestational diabetes: a population-based study in Alberta, Canada, 2005-11. J Diabetes 2016;8:45-55.

25 Statistics Canada. Table 051-0001 - Estimates of population, by age group and sex for July 1, Canada, provinces and territories, annual (persons unless otherwise noted), 2018. Available: http://www5. statcan.gc.ca/cansim/a26? lang=eng\&id $=510001$

26 Walker JD. Nice guidance on diabetes in pregnancy: management of diabetes and its complications from preconception to the postnatal period. NICE clinical guideline 63. London, March 2008. Diabet Med 2008;25:1025-7.

27 Ekoé J-M, Punthakee Z, Ransom T, et al. Screening for type 1 and type 2 diabetes. Can J Diabetes 2013;37 Suppl 1:S12-15.

28 Persson M, Razaz N, Tedroff K, et al. Five and 10 minute Apgar scores and risks of cerebral palsy and epilepsy: population based cohort study in Sweden. BMJ 2018;360:k207.

29 Austin PC. Using the standardized difference to compare the prevalence of a binary variable between two groups in observational research. Commun Stat Simul Comput 2009;38:1228-34.

30 Zou G. A modified poisson regression approach to prospective studies with binary data. Am J Epidemiol 2004;159:702-6.

31 Schoen C, Navathe R. Failed induction of labor. Semin Perinatol 2015;39:483-7.

32 Hiersch L, Borovich A, Gabbay-Benziv R, et al. Can we predict successful cervical ripening with prostaglandin E2 vaginal inserts? Arch Gynecol Obstet 2017;295:343-9.

33 Prysak M, Castronova FC. Elective induction versus spontaneous labor: a case-control analysis of safety and efficacy. Obstet Gynecol 1998;92:47-52

34 Grobman WA, Rice MM, Reddy UM, et al. Labor induction versus expectant management in low-risk nulliparous women. $N$ Engl J Med 2018;379:513-23.

35 Bell R, Bailey K, Cresswell T, et al. Trends in prevalence and outcomes of pregnancy in women with pre-existing type I and type II diabetes. BJOG 2008;115:445-52.

36 American Diabetes Association. (12) management of diabetes in pregnancy. Diabetes Care 2015;38 Suppl:S77-9. Suppl:

37 Murphy HR, Bell R, Cartwright C, et al. Improved pregnancy outcomes in women with type 1 and type 2 diabetes but substantial clinic-to-clinic variations: a prospective nationwide study. Diabetologia 2017;60:1668-77. 